\title{
GILBERTO PORRETANO: COMENTARIO AL TRATADO DE BOECIO SOBRE LA PREDICACIÓN SUSTANCIAL DE LOS NOMBRES DE LAS PERSONAS DIVINAS
}

\author{
David Torrijos Castrillejo \\ Universidad Eclesiástica San Dámaso (Madrid)
}

\begin{abstract}
Gilberto Porretano desempeña un papel cardinal en la recepción de Boecio en la Edad Media. Es el gran comentador de sus obras teológicas en las cuales se trata de bosquejar - por así decir - algo equivalente a un nuevo Órganon para las ciencias sagradas. Precisamente por esta razón es grande el interés que revisten tales obras de Boecio así como sus comentarios de Gilberto, también para la filosofía. Es notoria, por ejemplo, la introducción en los comentarios del Porretano de la célebre distinción entre la esencia como quo est y el sujeto o sustrato como quod est, que está a la base de la distinción entre acto de ser y esencia en las reflexiones de san Alberto Magno y santo Tomás de Aquino. ${ }^{1}$ En el opúsculo que presentamos esta distinción se halla plenamente operante.
\end{abstract}

El escrito en cuestión es conocido hoy en día como el segundo libro del Comentario al tratado sobre la Trinidad de Boecio, cuya primera parte es mucho más extensa. Sin embargo, ha circulado también como una obrita aparte bajo el título De predicatione. Su objetivo es elucidar si deben predicarse «sustancialmente» los nombres referentes a las personas divinas en cuanto tales («Padre», «Hijo», «Espíritu Santo»), así como los términos que expresan las peculiaridades de éstas («paternidad», «filiación», etc.). La cuestión que está en juego es hacer justicia a la fe cristiana que confiesa tres sujetos personales en Dios, a la vez que se respetan las verdades adquiridas a través de la lógica. No cabe duda que Dios es una sola sustancia, una sola realidad. Sin embargo, este ser se predica de tres personas. Ahora bien, ¿estos sujetos son también predicables de la divinidad?, ¿constituye un predicado sustancial el nombre propio de tales personas? Si se dice que «el Padre es Dios», «el Hijo es Dios», etc. y, a la vez, sólo existe una sustancia divina, entonces, ¿qué valor entitativo poseen los términos que enuncian los tres sujetos divinos? La solución que la Tradición eclesiástica ha dado a este problema ha sido el recurso al concepto de «relación», puesto que los nombres de «Padre», «Hijo» y «Espíritu santo» hacen referencia a determinadas relaciones entre las tres personas divinas. Ahora bien, ¿cuál es la naturaleza de esta relación? Aquí es donde entra una peculiaridad de la obra del Porretano (nn. 59ss.).

Evidentemente, aquello que Gilberto pretende evitar es afirmar que cada una de las personas divinas sea una sustancia divina distinta y así introducir división en Dios. Por ello

1 Me referí someramente a ello en mi introducción a San Alberto Magno, Introducción a la metafísica. Paráfrasis al primer libro de la Metafísica de Aristóteles, D. Torrijos Castrillejo (ed.), Madrid, Ediciones Universidad san Dámaso, 2013, pp. 1-liii, especialmente notas 137.145. Sobre esta cuestión, puede consultarse: NashMarshall, S. «Boethius's Influence on Theology and Metaphysics to C.1500», en N. H. Kaylor, Jr. and P. E. Phillips (eds.), A Companion to Boethius in The Middle Ages, Leiden, Brill, 2012, pp. 188-190. 
recurre a la relación, pero lo hace como una propiedad accidental respecto de la esencia divina. Además, la considera como algo extrínseco a ella. Así, la paternidad, la filiación y la unión (connexio), es decir, los aspectos por los cuales cada una de las personas divinas se distinguen, serían adventicios respecto a la única sustancia divina. De tal modo se preserva una única esencia pero se multiplicarían las personas en virtud de las relaciones, tal como ya apuntaba Boecio. Sin embargo, la interpretación del Porretano dejaría las relaciones fuera de la esencia divina y, por lo tanto, estaríamos próximos a las posturas modalistas así como, en general, aquellas herejías que comprendían las personas divinas como distintas maneras de presentarse el único Dios (algo contrario a la doctrina de la fe enunciada en los Concilios de Nicea y Constantinopla). Éste es un error en el que cayó de modo claro Abelardo, también a causa de un inapropiado uso de la lógica y, de hecho, es reprobado en nuestra obra (n. 30).

Santo Tomás de Aquino ( $S . T h$. I, 28, 2, in c.) se refiere al fallido intento del Porretano al exponer la doctrina trinitaria, del cual se habría retractado en el Concilio de Reims. ${ }^{2}$ El problema de su exposición habría estribado en una deficiente comprensión del accidente de la relación. En cierto modo - admite el Aquinate-, tiene razón Gilberto (nn. 3-4.61) al decir que, mientras la cantidad y las cualidades se predican de modo intrínseco debido a una alusión directa a la esencia de la cosa, la relación se refiere en todo caso a algo distinto de dicha esencia. En este sentido, la relación sí se asemeja a los accidentes «extrínsecos» que, como el lugar, el hábito predicamental, etc., hacen referencia explícita a una realidad distinta de la esencia de la cosa en la cual inhieren. Sin embargo, objeta santo Tomás, las relaciones no están propiamente sobreañadidas a las esencia de modo extrínseco sino intrínsecamente, es decir, se trata de algo que afecta auténticamente al ser de la cosa, si bien de modo accidental. No obstante, en Dios no se puede admitir la presencia de accidentes, luego, si admitimos una relación, ésta ha de ser idéntica con la sustancia divina. Estas relaciones no supondrán una vinculación con algo externo al ser divino, sino que expresarán la oposición entre una persona y otra, siempre internamente al ser divino.

Otra inexactitud que santo Tomás (S. Th. I, 39, 4, in c.) recrimina a Gilberto es su modo de comprender el nombre «Dios» como idéntico con la divinidad o «deidad» (Pról. 2; nn. 1.45.55), como si de un término genérico se tratara. De esta manera, dicho nombre indicaría propiamente la esencia divina pero tan sólo podría emplearse como sujeto - en lugar de los nombres de las tres personas-, valiéndose de una añadidura conceptual. Ciertamente, Gilberto trata la naturaleza divina con los mismos patrones con que se suele hablar de los entes compuestos, para los cuales puede caber una multiplicidad de individuos específicamente idénticos. Así, para designar la naturaleza humana individualizada en un sustrato no vale mencionar la «humanidad» (quo est) sino a «un hombre» (quod est), el cual es el único que puede ser tomado como sujeto. Por tanto, no se dirá «la humanidad está sentada» sino «este hombre está sentado». Esto mismo, algo bien razonable en el hombre, también es aplicado a Dios. Por tal motivo, parece que en el Concilio de Reims Gilberto afirmaba que «cuando se

2 En el Símbolo formulado ante san Bernardo leemos esta confesión dirigida contra Gilberto, la cual es la que mejor cuadra con la objeción puesta aquí por santo Tomás: «Creemos que un solo Dios, Padre, Hijo y Espíritu santo es eterno y ninguna cosa hay en Dios - díganse relaciones, propiedades, singularidades o unidades-que sea eterna y no sea Dios» (PL 185, 618A = Dz 391, ausente en DH). San Bernardo, por su parte, nos proporciona la noticia de su retractación: «Pero no digamos ya todo esto contra quien, en el mismo Concilio, aceptando humildemente las palabras de los obispos, rechazó con sus propios labios tanto éstas como las demás proposiciones halladas dignas de reproche»; Bernardus Claraevallensis, In Cantica canticorum, LXXX, 9; Sermones super cantica canticorum, J. Leclercq, C.H. Talbot et H.M. Rochais (eds.), vol. 2, Bernardi opera, Romae, Ed. Cistercienses, 1958, p. 283; PL 183, 1170D. 
dice «Dios», uno no se refiere a la sustancia que es (quae est) sino por la que es (qua est)». ${ }^{3} \mathrm{El}$ término «Dios» aludiría a la esencia y no debería ser tomado propiamente como sujeto por no ser un sustrato, a diferencia de las personas divinas. Vemos como la célebre distinción entre sustrato y esencia (quod est y quo est) no fue utilizada con plena destreza por nuestro teólogo e incluso le valió una condenación. ${ }^{4}$

Por su parte, Tomás piensa que en Dios los nombres que se identifican con su propia esencia no tienen por qué ser proferidos de modo genérico sino también dando por supuesto la subsistencia actual en el sustrato. En Dios, al hablar de «divinidad» no se procede del modo seguido cuando se menciona en el hombre su «humanidad», porque en Aquél se da por sobreentendido en el término que esa esencia subsiste actualmente en una sola sustancia y, a la vez, en tres personas ( $S$. Th. I, 39, 4, ad 3).

Estas imperfecciones en la exposición de la doctrina de Boecio no obstan para que Gilberto haya acertado en lo fundamental, a saber, que los nombres de «Padre», «Hijo» y «Espíritu santo» no se predican de un modo idéntico a como lo hacen los atributos divinos idénticos con su esencia (como «uno», «infinito», «omnipotente», etc.). En definitiva, la reflexión sobre la Trinidad divina se revela un tema de gran fecundidad no sólo para la teología sino también para el desarrollo y la sutil profundización de la filosofía.

\section{BOECIO}

\section{Al diácono Juan de la Iglesia de Roma, sobre la cuestión de si los nombres Padre, Hijo y Espíritu Santo se predican de la divinidad sustancialmente ${ }^{5}$}

1. [1299] [SRT32] [M182] Me propongo investigar si «Padre», «Hijo» y «Espíritu santo» se predican de la divinidad sustancialmente o de algún otro modo. Creo que el derrotero de la investigación debe [1300] ser tomado de allá donde consta el inicio manifiesto de estos asuntos, esto es, de los fundamentos mismos de la fe católica. Así pues, cuando pregunto si lo que se llama «Padre» es sustancia, [1301] se me responde que es sustancia. Cuando pregunto si el Hijo es sustancia, se me dice lo mismo. Igualmente, nadie dudará que también el Espíritu santo es sustancia. Ahora bien, al seguir pensando que el Padre, [M183] el Hijo y el Espíritu santo no son varias sino una sola sustancia, entonces una sustancia de tres no se puede separar en modo alguno ni ser disgregada, ni tampoco ha sido compuesta a partir de tres partes, sino

3 Gaufridus Claravallensis, Epistola ad Albinum. De condemnatione errorum Gilberti Porretani, 4; PL 185, 589D. Cf. Bernardus, o.c., 8; Leclercq et al. (eds.), vol. 2, p. 282; PL 183, 1170C-D; De consideratione, V, 7 , 15; Leclercq et al. (eds.), vol. 3, p. 479; PL 182, 797A-B.

4 Gilberto «iguala el sentido de «sustancia» con el Boeciano id quod est (aquello que es) y el de su «cualidad» con el id quo est (aquello por lo cual el quod est es). También explica que, en una proposición dada, sólo funciona uno de estos dos sentidos: en la posición de sujeto, el nombre significa sólo la sustancia (es decir, posee un significado denotativo), mientras en la posición de predicado significa sólo la cualidad. Esta posición fue considerada típica de la escuela porretana fundada por Gilberto y que produjo textos sobre lógica y gramática»; I. Rosier-Catach, «Grammar», en R. Pasnau and Ch. van Dyke (eds.), The Cambridge History of Medieval Philosophy, vol. 1, Cambridge, Cambridge UP, 2010, pp. 199-200.

5 Sigo la edición crítica de Boethius, De consolatione philosophiae. Opuscula theologica, C. Moreschini (ed.), Monachii, Saur, 2005, pp. 182-185. Cf. Boethius, The theological tractates. The consolation of philosophy, The Loeb Classical Library, H.F. Stewart, E.K. Rand, S.J. Tester (eds.), Cambridge, Harvard UP, 1973, pp. 32-36. Las páginas de la versión de estos dos últimos trabajos van precedidas de las iniciales de sus respectivos editores. También hago referencia a las columnas de Migne: PL 64, 1299-1302. 
que es absolutamente una. Cualesquiera afirmaciones que se digan de la sustancia divina han de ser comunes para los tres. Será signo de esto que, cualquier cosa que se predique [SRT34] de la sustancia divina, todo aquello que se diga de algún modo acerca de cada uno de ellos tres, reunidos en uno, será predicado de los tres singularmente. De esta manera, si decimos: «el Padre es Dios, el Hijo es Dios, el Espíritu santo es Dios»; el Padre, el Hijo y el Espíritu santo son un solo Dios. Así pues, si su deidad, una sola, es una sola sustancia, el nombre de «Dios» puede predicarse sustancialmente de la divinidad.

2. Así, el Padre es verdad, el Hijo es verdad, el Espíritu santo es verdad: el Padre, el Hijo y el Espíritu santo no son tres verdades sino una sola verdad. Así pues, si existe una sola sustancia en ellos, habrá una sola verdad: es necesario que la verdad se predique sustancialmente. Sobre la bondad, la inconmutabilidad, sobre la justicia, la omnipotencia y sobre todos los restantes predicados que hacemos tanto de cada uno como de todos ellos, es evidente que ha de hablarse de modo sustancial. Según esto, se ve que aquello que conviene sea dicho en cada uno por separado, no se puede empero decir en todos, no se predica sustancialmente, sino de otro modo; más tarde investigaré cuál modo sea éste. En efecto, quien es Padre no transmite tal vocablo al Hijo ni al Espíritu santo. Por lo cual sabemos que [M184] dicho nombre no pertenece a la sustancia, pues si fuera de carácter sustancial, tal como sucede con «Dios», «verdad», [1302] «justicia» y el nombre mismo de «sustancia», también se diría de los otros.

3. Igualmente, tan sólo el Hijo recibe tal nombre, ni se une a los otros como «Dios», «verdad» y los demás a los que me referí antes. El Espíritu santo no es el mismo que el Padre y el Hijo. Por esto entendemos que «Padre», «Hijo» y «Espíritu santo» no se predican de la divinidad misma sino de otro modo. [SRT36] Si se predicasen de modo sustancial, se referirían tanto a cada uno como a todos en singular. Pero es evidente que estos nombres se dicen en relación a alguien, pues el Padre es padre de alguien, el Hijo es hijo de alguien y el Espíritu es espíritu de alguien. De esto resulta que tampoco se predica de Dios la trinidad de modo sustancial. En efecto, el Padre no es trinidad, pues quien es Padre no es Hijo ni Espíritu santo. Del mismo modo, tampoco el Hijo es trinidad ni el Espíritu santo es trinidad, sino que la Trinidad consiste en la pluralidad de personas, la Unidad en cambio en la simplicidad de la sustancia.

4. Así, si las personas están divididas pero la sustancia es indivisa, es necesario que el término que toma su origen de las personas no pertenece a la sustancia. Sin embargo, la Trinidad produce la distinción de las personas, luego la Trinidad no pertenece a la sustancia. De esto resulta que ni el Padre, ni el Hijo, [M185] ni el Espíritu santo, ni la Trinidad se dicen de Dios sustancialmente, sino - como se dijo - en relación a alguien. «Dios» empero, así como «verdad», «bondad», «omnipotencia», «sustancia», «inmutabilidad», «poder», «sabiduría»y todo lo que de tal modo pueda pensarse se dice sustancialmente de la divinidad. Te ruego me aclares si todo esto está dicho correctamente y según la fe, o bien, si opinas de modo diverso en algún aspecto, examines atentamente lo dicho y pongas de acuerdo la fe y la razón en la medida de lo posible. 


\section{GILBERTO PORRETANO}

\section{COMENTARIO DEL SEGUNDO LIBRO DE BOECIO SOBRE LA TRINIDAD (ACERCA DE LA PREDICACIÓN DE LAS TRES PERSONAS) ${ }^{6}$}

\section{Prólogo}

1. [1301] [H161] Gracias a la investigación de aquella cuestión sobre la cual Boecio escribió a Símaco, quedaron bien de manifiesto los predicamentos de las cosas naturales con sus distintos géneros y razones. Algunas de estas razones se pueden traspasar en cierta medida a los estudios teológicos y predicarse también de Dios, tal como se hace respecto de los seres subsistentes: unas, por sí mismas, otras, en cambio, por comparación de una realidad con otra realidad diferente. Entre las razones predicadas por sí, unas son predicadas en cuanto definitorias de la cosa, otras en cuanto definitorias de las circunstancias. Tales son también todas aquellas que son predicadas por comparación.

2. Porque, sin embargo, algunos de escaso [1302] entendimiento, oyendo que Dios es simple, Lo consideran a Él y a cualesquiera de los variados nombres que se pronuncien acerca de Él - como, a saber, «Dios», «uno», «eterno», «persona», «principio», «autor», «Padre», «Hijo», «conexión», etc. - de la misma naturaleza y razón que la esencia por la cual se dice que Dios es ser, que la unidad por la cual es uno, que la eternidad por la cual es eterno, etc. Y viceversa, el Padre mismo sería la paternidad, el Uno, la unidad y el Eterno, la eternidad. Se procede del mismo modo para todos los predicados que [1303] se hagan de Él por la razón que sea. Boecio, por su parte, escribe al diácono romano Juan especialmente sobre aquellos predicados que se hacen con los nombres de «Padre», «Hijo» $\mathrm{y}$ «Espíritu santo».

3. Muestra que éstos se predican de sujetos distintos y que son distintos no sólo entre sí sino incluso respecto de la esencia que, siendo una sola, se dice con diversos nombres acerca de aquellos mismos sobre los cuales se hacen también esos diversos predicados; todo ello sin detrimento de la simplicidad de Dios.

4. Ciertamente, tal distinción parece, o no poder ser mostrada en absoluto, o sólo poder ser manifestada con esfuerzo. Sea porque la especulación teológica [H162] no admite completamente las leyes naturales, sea porque - como se ha dicho- el error de los escasos de entendimiento repugna cualquier nombre que signifique diversidad en el Dios simple, Boecio no trata de persuadir sobre esta distinción con testimonios abundantes ni tomados de escritos autorizados, como trabajosamente suele hacerse en este asunto, a causa de su dificultad así como por su oscuridad; tampoco prueba mostrando las conexiones de los hallazgos necesarios, ni se explica alargándose con las controversias sobre aquello que pudiera surgir incidental y como colateralmente. Por el contrario, dado que su interlocutor no es malévolo ni torpe, procede en su demostración valiéndose tan sólo de la razón por la que se predica propiamente cada cosa.

5. Muestra con bastante evidencia la propiedad de aquella predicación según la cual, si se afirma acerca de un sujeto uno de dos predicados, el otro no puede ser también afirmado;

6 Sigo el texto y la numeración de los párrafos correspondiente a The Commentaries on Boethius by Gilbert of Poitiers, N. M. Häring (ed.), Toronto, Pontifical Institute of Mediaeval Studies, 1966, pp. 159-180. Indico las páginas de ese volumen antecedidas de la inicial del editor. En Migne (PL 64, 1301-1310), cuya numeración también figura en nuestra traducción, recibe el título: Gilberti Porretae Commentaria in Librum de Praedicatione Trium Personarum. 
éstos no sólo son distintos entre sí, sino también lo son respecto de todo aquello que se diga en común de sus sujetos y son distintos principalmente respecto de aquel único nombre de entre los tres que se predican sustancialmente.

6. Esta distinción no consiste en el número (por el cual esto es uno, mientras que cualquier otra cosa es una), sino en la naturaleza del género y en lugar de la razón. Ahora bien, callando respecto de la distinción numérica y de la distinción según el género, se ocupa en primer lugar de aquella que es según razón; hacia el final, se acuerda de aquella distinción según el género que sigue necesariamente a la distinción numérica.

\section{Exposición del texto}

\section{I}

1. [H163] Así pues, igual que en un teatro de sofistas, como alguien verdaderamente cierto adaptándose a quienes albergan dudas, dice: ME PROPONGO INVESTIGAR SI EL PADRE, EL Hijo y el Espíritu santo..., esto es, cuáles de estos nombres que significan lo que, con diversas razones, los gramáticos denominan «cualidades», los dialécticos, «categorías» esto es, predicamentos-, se prediquen sustancialmente; es decir, si, de acuerdo con la proporción de aquellas cosas que, entre las realidades naturales, son denominadas «ser de las cosas subsistentes», también SE PREDICAN aquellos nombres DE LA DIVINIDAD, o sea, de aquellos sujetos que, siendo lo que son sólo en virtud de la divinidad, si bien no se denominan «Dios», sí se llaman «divinidad»: se ha de ver si no se predican sustancialmente sino DE OTRO MODO.

2. Añade a esto: ALGÚN, porque no existe un solo modo de predicar cuando se está tratando de las realidades naturales, desde las cuales se ha trasladado a la teología la costumbre de usar estos términos partiendo de la proporción de cierta razón.

3. En efecto, de entre aquellos aspectos que no pertenecen al ser de algunos, algunos se entienden por sí mismos, es decir, no es menester comparación alguna: tal como lo blanco no se denomina blanco por referencia a nada; otros aspectos, por el contrario, se predican de manera que, en virtud de su predicación misma, los sujetos de los cuales se predican son referidos a otros: tal como el lugar, por el cual algo se dice estar «más allá»; el tiempo, por el cual se dice que algo es «de hoy»; el hábito, por el cual se dice que «lleva casco»; la relación, por la cual se dice que es «tal» 0 «tanto».

4. Gracias a todas estas cosas, se sobreentienden otras. Además, de éstos, unos se dicen según la cosa misma, como el blanco; otros, son añadidos extrínsecamente, como el lugar y los demás que se han dicho antes, así como los pertenecientes a los restantes géneros.

5. [H164] De estos y de otros modos que pueden ser mostrados siguiendo otras divisiones, se dicen muchos predicados de las cosas subsistentes. Así pues, cuando esto se transfiere a la teología, también el modo de predicar será múltiple.

\section{CReo Que El DERrotero, etc.}

Como si dijese: igual que haría un sofista, me propongo investigar si tales cosas antes mencionadas sobre Dios, o sea, sobre aquello que constituye un solo Dios y también una sola divinidad, se predican sustancialmente o bien de otro modo y, en tal caso, de cuál. Como quien demuestra competentemente cuál de estas cosas sea verdad, me apresuro a investigarlo con ahínco.

Creo que el derrotero de la investigación debe Ser tomado de allá donde CONSTA el INICIO MANIFIESTO (o sea, donde manifiestamente es firme el inicio) DE ESTOS ASUNTOS, ESTO ES, DE LOS FUNDAMENTOS MISMOS DE LA FE CATÓLICA. 
7. En las demás disciplinas, en las cuales siempre se acomodan regularmente la generalidad y la necesidad, [1304] la razón no sigue a la fe, sino la fe a la razón. Puesto que en las cosas temporales no existe nada que no esté sometido a cambio, toda la necesidad de tales cosas, acomodada a la costumbre, vacila.

8. En efecto, en esas cosas, todo lo que se predica como ser necesario o no ser necesario, en cierto modo, ni es necesariamente ni no es necesariamente. En efecto, no es absolutamente necesario aquello a lo cual únicamente la costumbre acomoda el nombre de «necesidad».

9. En las cuestiones teológicas, por el contrario, donde existe la necesidad absoluta y propiamente dicha, no es la razón quien antecede a la fe, sino la fe antecede a la razón. En dichas cuestiones, pues, no poseyendo conocimiento, creemos, pero conocemos al creer; porque, prescindiendo de los principios de la razón, la fe concibe no sólo unos principios tales que las razones humanas no son capaces de entender, sino también los que permiten a éstos ser principios.

10. En efecto, el Espíritu que procede de Dios proporciona a la fe esta dignidad superior a las razones tanto para los asuntos teológicos como para aquellas cuestiones inferiores a las teológicas, [H165] como las cuestiones naturales y las demás, cuya credibilidad fue atribuida por el espíritu de este mundo a las razones de los filósofos.

11. Tanto en las cuestiones naturales cuanto en otras, la fe de las realidades espirituales precede a toda la razón de modo que todas las cosas sean juzgadas sobre todo por la fe, antes que por la razón. También por esto se dice correctamente que la fe católica es el inicio no sólo de las cuestiones teológicas sino también de todas las cosas susceptibles de ser entendidas; «inicio», es decir, fundamento que no vacila con incerteza alguna e incluso sostiene certísima y firmísimamente las cosas mudables.

12. Partiendo de este inicio o fundamento, comenzando el derrotero de la antedicha investigación, dice: Así PUES, CUANDO PREGUNTO, etc.

Preguntó si, de aquellos sujetos de los cuales se dicen los términos «Padre», «Hijo»y «Espíritu santo», éstos se predican sustancialmente o de algún otro modo. Una cuestión que no es simple sino que está compuesta de varias cuestiones.

13. Con estas palabras tocantes a esos tres sujetos se entiende que ha formulado distintas cuestiones: primero, si se predican sustancialmente o no se predican sustancialmente, sino de otro modo distinto de la predicación sustancial; después, en tal caso, de qué otro modo. Pero parece que debía haber buscado primero si se predican o no. Esto es algo que podría haber buscado con toda razón, porque, entre todas las cosas, hay unas que se predican y otras que no. Además, si constase que no se predican, no quedaría nada por ser inquirido. Ahora bien, si constase que se predican, entonces estaría en razón investigar cómo se predican, a saber, si sustancialmente o de otro modo. Por fin, si no es sustancialmente, sino más bien de otro modo, quedaría por buscar de qué modo.

14. Boecio, por su parte, reúne la segunda cuestión y la tercera en una sola, dejando pasar la primera, dado que para todos es cierto que se predican aquellos términos a partir de los cuales se produce la denominación trina de estos nombres. Pero las divide en una investigación por la cual suprime la duda de ambas.

15. En primer lugar, qué haya de sentirse acerca de la cuestión por la cual se interroga si se predican sustancialmente o no, es elucidado por Boecio valiéndose de aquel razonamiento [H166] que Cicerón llama «conclusión simple». Éste suena así: lo que se predique sustancialmente sobre Dios, se dice también del Padre, del Hijo y del Espíritu santo, tanto separadamente de cada uno, como a la vez de todos. 
16. Ninguno de los tres términos - a saber, «Padre», «Hijo»y «Espíritu santo»— sobre los cuales se habla aquí, se dice de todos, ni por separado, ni a la vez. Luego ninguno de ellos se predica sustancialmente.

17. La primera parte de este razonamiento - que los dialécticos denominan «proposición» $\mathrm{o}$ «presupuesto» y los rétores «exposición»- es confirmada por la fe católica. Tomando, PUES - dice-, de esta sentencia el derrotero de la investigación: CUANDO PREGUNTO SI aquello QUE SE LLAMA «PADRE» ES, según la $u s y a m^{7}$ de su género, SUSTANCIA, esto es, ente o subsistente, SE ME RESPONDE, por la autoridad de la fe católica, que es verdaderamente SUSTANCIA.

18. CUANDO PREGUNTO SI aquel que se denomina «HIJO» ES SUSTANCIA, por la autoridad de la misma fe católica, SE ME DICE LO MISMO, es decir: es SUstancia. IGUALMENTE, NADIE que posea la fe católica DUDARÁ - es decir, no podrá dudar- QUE TAMBIÉN EL ESPÍRITU SANTO ES SUSTANCIA.

19. Así, pues, el término «sustancia» se predica por sí de cada uno de los tres y también por separado. Sin embargo, no se dice la «sustancia» de cada uno separadamente SINO QUE se predica conjuntamente de los tres la misma «sustancia». Al SEGUIR PENSANDO, pues, suponiendo en virtud de la misma fe, que el PADRE, el HIJO y el ESPÍRITU SANTO NO SON VARIAS cosas puestas en el número de las esencias, SINO que ES SUSTANCIA numéricamente UNA, en la singularidad de una sola esencia, [1305] sustancia por la que se dice que aquéllos son.

20. Es ENTONCES manifiesto que hay UNA sola SUSTANCIA para estos tres, la cual se llama en griego usya, pero en latín, como se ha dicho, substantia (sustancia), subsistentia (subsistencia) o - para decirlo más claramente- essentia (esencia). Ésta NO SE PUEDE SEPARAR EN MODO ALGUNO NI SER DISGREGADA, NI TAMPOCO HA SIDO COMPUESTA A PARTIR DE TRES PARTES.

21. [H167] Se dice de la esencia que es «una en la singularidad o en la propiedad de la cosa que se predica de ellos». Esto debe ser entendido no en la unión de cosas diversas, por la cual sucede con frecuencia que muchas realidades son denominadas una «unidad». Esto sucede, en primer lugar, debido a cierta conformidad, por la cual varios hombres son denominados «un hombre»; porque, aquellos cuyas subsistencias podrían ser denominadas «humanidades» por los matemáticos, siendo hombres distintos por la diversidad numeral de éstas, son semejantes según una sola conformidad y son conformes por semejanza. También sucede, en segundo lugar, por la integridad, por la cual el cuerpo y el alma son denominados «un animal», no porque aquellas realidades de las cuales se compone el animal son o pueden ser «animal»-pues nunca las partes de algo son en virtud de toda la forma del todo-, sino porque el animal mismo que está constituido por ellas es animal en virtud de la forma compuesta de la totalidad de tales subsistencias, a saber, de esa alma y de ese cuerpo. Además, cada una de las partes singulares se predican en singular de él, que está formado de partes sin confusión con otro o con cada una de las dos partes. De esto se hablará más extensamente en la exposición del libro escrito Contra Eutiques. ${ }^{8}$

22. Existen también otras uniones de otras realidades diversas unidas entre sí en virtud de sus propiedades, las cuales están vinculadas de otras maneras. Ahora bien, para que la explicación respondiera a la naturaleza de esta obra, callando sobre las demás, procuramos recordar tan sólo éstas, las cuales fueron rechazadas de la divina esencia por el autor, al parecer, contra los errores de algunos.

7 Gilberto escribe usyam: se refiere, naturalmente, a la ov̉øí $\alpha$ aristotélica: cf. n. 20.

8 Cf. Contra Euticen et Nestorium, IV, 14-36; The Commentaries on Boethius by Gilbert of Poitiers, Häring (ed.), pp. 290-294. 
23. [H168] En efecto, algunos herejes cuyo error recuerda Epifanio sin mencionar a su autor, dicen que el Hijo es «semejante en la esencia»" de aquel que también es Padre. En este punto en parte concuerdan con los arrianos, en parte difieren de ellos. Por esta razón son denominados semiarrianos.

24. Ciertamente, los arrianos - con los cuales conviene Eunomio-niegan que el Padre y el Hijo sean de una sola esencia, ni por unión de sus diversas esencias, ni por la singularidad de una sola esencia. Dado que una criatura no puede ser semejante a su principio, piensan que el Hijo - quien, según su opinión, es criatura bajo todos los aspectos de su naturaleza-es completamente desemejante del Padre.

25. Estos semiarrianos niegan que el Hijo y el Padre sean de una sola sustancia según la singularidad de una sola esencia, pero afirman que sí lo son según la conformidad de diversas esencias. Así pues, en cierto modo disgregan y separan esta sustancia que es la esencia del Padre y del Hijo, la cual es entendida según una singularidad, al pensarla como una en la unión de una semejanza. Tal como son distintas aquellas cosas que son semejantes, también han de serlo aquellas otras en cuanto son a su vez semejantes.

26. Otros herejes, que igualmente recuerda Filastrio, afirman que Dios es triforme de tal modo "que cierta parte suya es el Padre, otra el Hijo, otra el Espíritu santo: esto es, que las partes del Dios uno forman esta Trinidad, como si Dios se completase a partir de estas tres partes y no fuera perfecto en sí ni el Padre, ni el Hijo, ni el Espíritu santo».10

27. De acuerdo con este error, algunos, al leer que el Hijo está en el Padre,$^{11}$ interpretaron esto como un vaso menor se encuentra en un vaso mayor. Por esto, Filastrio ${ }^{12}$ los denomina «Metangismonitas». ${ }^{13}$ Pues el vocablo griego angismon significa vas (vaso) en latín y metangismon, por su parte, la entrada de un vaso en otro. ${ }^{14}$

28. Éstos, de acuerdo con su punto de vista, unen tal sustancia - esto es, la esencia de Dios - formando una sola cosa como si estuviera partida. Efectivamente, si Dios estuviera compuesto en cierto modo de partes, también su esencia, por la cual Él es, estaría compuesta de aquellas esencias que son partes de Dios. [H169] Siendo así que un subsistente está compuesto de subsistentes, es necesario que constituya un todo, es decir, que aquella subsistencia por la cual es perfecto esté compuesta de todos los subsistentes de todas sus partes.

29. Sin embargo, no decimos a la inversa que, siempre que el ser de cierta entidad esté compuesto de varias cosas, también aquello mismo que [1306] esa entidad es, estará compuesto de muchos. En efecto, sucede que un subsistente puede ser simple, o sea, de ningún modo compuesto de subsistentes, siendo empero múltiple su ser: por ejemplo, tal como el alma es simple. Sin embargo, también hay algo con muchos subsistentes.

30. De tal manera también algunos, opinando que Dios era multiforme, atribuyen al Dios uno y simple la potencia, la sabiduría y la bondad como realidades diversas. Afirman que Dios, siendo uno y el mismo, es, según la potencia Padre, según la sabiduría Hijo y según la

9 Epifanio según Augustinus, De haeresibus, 51; CC 46, 322; PL 42, 39. Gilberto cita la obra de Epifanio a través del tratado Sobre los herejes de san Agustín, donde éste confiesa su dependencia de la obra de aquel: cf. ibid., Praefatio, 6; CC 46, 288; PL 42, 23.

10 Ibid., 74; CC 46, 335; PL 42, 45.

11 Cf. Jn 14, 10.20.

12 Cf. Filastrius, Diversarum heresion liber, 51; CC 9, 238-239. Gilberto también parece conocer a Filastrio también a través de Agustín: cf. De haeresibus, 41; CC 46, 309; PL 42, 33.

13 Cf. Filastrio según Augustinus, De haeresibus, 58; CC 46, 327; PL 42, 41.

14 Los términos griegos que aparecen en la edición del Corpus christianorum de san Agustín son, respec-

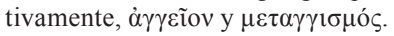


bondad Espíritu santo; hablan valiéndose, pues, no tanto de argumentos cuanto de artimañas - si bien frágiles como el vidrio $-{ }^{15}$

31. A todos éstos los destruye cuando dice: «No se puede separar en modo alguno ni ser disgregada» aquella esencia una que pertenece a tres, por la cual éstos son. «Ni tampoco ha sido compuesta a partir de tres partes», SINO más bien ES ABSOLUTAMENTE UNA aquella por la cual los tres son uno y simple y son absolutamente aquello que son. En efecto, no hay otra cosa por la cual sean excepto aquella esencia simple e individual. En virtud de ésta se predican acerca de los tres en común, tanto por separado, como colectivamente, tal como fue dicho.

32. Aquello que se diga DE LA SUSTANCIA DIVINA, es decir, que se predique sustancialmente acerca de Dios, eso ha DE SER común PARA LOS TRES, o sea, para el Padre, el Hijo y el Espíritu santo.

De tal manera, hubo de decir en singular «aquello que» $\mathrm{y}$ «se diga» así como «eso» $\mathrm{y}$ «común» porque, como se dijo, no hay sino una sola cosa que se diga sustancialmente de ellos.

33. Ahora bien, sin embargo, eso se muestra frecuentemente con proporciones de ciertas razones y con diversas maneras de significar, esto es, sea en relación al género, sea en relación a una imitación de las cualidades o de las cantidades - como, por ejemplo, cuando decimos: «Dios es bueno y grande»- [H170] y del mismo modo se significa con otros nombres. Por esta razón, dijo en plural: CUALESQUIERA, SE DIGAN, HAN DE SER y COMUNES.

34. En las cuestiones teológicas hablamos usando semejanzas de las cosas, pues no podemos disponer de términos para aquellas realidades sobre las cuales hablamos; de tal modo, para significar éstas, traspasamos aquellas palabras que respectan a las restantes disciplinas en virtud de cierta proporción de razón. No resulta sorprendente que en tales cuestiones signifiquemos con muchos nombres y modos diversos lo mismo, pues es así que, en las cuestiones naturales, donde no existe tanta escasez de términos, procedemos de modo similar muchas veces: por ejemplo, «cuerpo», «corporal» y «corpóreo» significan lo mismo pero de un modo diverso e, igualmente, «animal» (como sustantivo) y «animal» (como adjetivo), así como «hombre»y «humano». Se podrían añadir otros muchos ejemplos.

35. SERÁ SIGNO DE ESTO, etc.

Es como si quisiera decir: cualquier cosa que se predique de la sustancia divina es común a los tres. SignO DE ESTO, es decir, este signo SERÁ aquel por el cual podrá entenderse probablemente LO QUE SON aquellas cosas QUE SE PREDICAN DE LA SUSTANCIA DIVINA, es decir, que se digan sustancialmente sobre Dios; TODO AQUELlo QUE, etc.

36. El haber dicho «signo» lo toma de los rétores. En efecto, Cicerón ${ }^{16}$ pone el signo entre aquellos hallazgos probables que normalmente suelen hacerse: gracias a él, aquello de lo cual el signo se dice, dícese que también es significado en cierto modo, pero necesita un testimonio mayor y una confirmación de mayor peso. ${ }^{17}$

37. De tal manera, también en este lugar de la predicación hecha acerca de los tres a la vez y acerca de cada uno en singular y por separado, la comunidad de esta misma sustancialidad se llama signo del predicado. Tal como «sangre», «huida», «desbandada», «polvo» y otros semejantes ${ }^{18}$ son signos de ciertos hechos que suelen suceder entre los que continuamente están ocupa-

15 Häring advierte que esta crítica está dirigida contra Abelardo y su escuela.

16 Cf. De inventione, I, 29, 46.

17 Cf. ibid., I, 30, 48 .

18 Cf. ibid., I, 38, 48. 
dos en empresas o bien en el desempeño de sus empresas, ${ }^{19}$ pero que, sin embargo, son de poco peso porque acontecen en contra de las tareas a las cuales se les atribuyen: de este modo esta sustancialidad de los predicados acerca de los tres por separado y conjuntamente constituye un cierto signo de los predicados porque esto conviene comúnmente con muchos predicados, pero resulta de poco peso porque algunas cosas se dicen por separado de cada uno y conjuntamente de los tres, tanto en la singularidad de la cosa como en la del nombre. Tales cosas no [H171] pueden ser propias de la sustancia de éstos pues no son predicamentos según su misma realidad sino añadidos extrínsecamente, como, por ejemplo, la «principalidad» y la «acción».

38. En efecto, también es por sí misma verdadera la proposición: «el Padre es principio»; e, igualmente, por sí misma verdadera: «el Hijo es principio»; y también es por sí verdadera: «el Espíritu santo es principio»; y, por fin, todos ellos a la vez: «el Padre, el Hijo y el Espíritu santo son un solo principio».

39. Del mismo modo, es verdadera por sí misma: «el Padre es hacedor»; también: «el Hijo es hacedor»; también: «el Espíritu santo es hacedor»; por último, a la vez: «el Padre, el Hijo y [1307] el Espíritu santo son un solo hacedor».

40. Tampoco la principalidad ni la acción constituye para ellos aquello por lo cual son lo que son, vale decir, su esencia. Mientras son por su esencia lo que son, en virtud de aquella relación denominada «principalidad» son referidos a las criaturas y son considerados «principio» de ellas. Ahora bien, a causa de la «acción» se dice que obran. Además, debe tenerse en cuenta que no admitieron el ser «principio», pero sí admitieron el «hacer».

41. Por todo esto se ve que las proposiciones acerca de estos tres en común concuerdan en lo tocante a cierto ser, el cual es predicado eternalmente, y también en lo relativo a otras afirmaciones hechas de modo temporal. Sin embargo, tales asertos tampoco se predican sustancialmente. Por consiguiente, la comunidad de los predicados constituye un signo de poco peso para revelar su sustancialidad.

42. Así pues, para demostrar aquello que se predica sustancialmente de Dios, ciertamente es menester emplear la comunidad de predicación, porque la predicación de todas las cosas sustanciales para los tres es realmente común. No obstante, tal sustancialidad necesita del testimonio de otra razón y de un lugar más sólido para confirmar lo hallado, pues no todo lo que se dice de ellos en común es necesario que se diga sustancialmente de ellos.

43. Por tanto, también este autor, cuando dijo «signo de esto» - esto es, de la comunidad de predicación- «será que cualquier cosa que se predique de la sustancia divina», no dijo QUE, cualesquiera sean los predicados que se hagan en singular acerca de los tres conjuntamente, se dicen de cada uno de ellos de tal modo, es decir, en singular - lo cual sería falso - sino más bien dice: Cualesquiera cosas Que Se Digan ACERCA DE CADA UNO DE Ellos [H172] por sí mismas, SE PREDICARÁN DE ESTE MODO - o sea, sustancialmente- también EN SINGULAR SOBRE LOS TRES CONJUNTAMENTE, es decir, tomados simultáneamente como sujetos.

44. Se confirma con los testimonios proporcionados por los ejemplos DE ESTA MANERA: SI, por ejemplo, DECimos por separado que «EL PAdRe es Dios» y también que «EL HiJo es Dios» así como «El Espíritu SANTO ES Dios», también diremos reuniéndolos que «EL PADRE, EL HiJo Y EL EsPíRITU SANTO SON Un SOlo Dios». Con este ejemplo se manifiesta que la «deidad» que se dice de cada uno por separado, se predica a la vez de ellos conjuntamente.

45. Así Pues, EXISTe una sola DeIDAd suya, es decir, del Padre, del Hijo y del Espíritu santo; SI ES - como de hecho es así- UNA SOLA aquella sustanCIA (en griego se dice $u s y a$, pero 
en latín essentia) a ellos perteneciente, entonces es manifiesto que EL NOMBRE DE Dios, es decir, el nombre de la deidad, que es «Dios», PUEDE PREDICARSE SUSTANCIALMENTE DE LA DIVINIDAD, o sea, de Dios, a saber, del Padre, del Hijo y del Espíritu santo.

46. Así, es decir, tal como dijimos que la deidad, la cual es predicada sustancialmente, se atribuía tanto a cada uno por separado como a los tres conjuntamente, igualmente decimos que la verdad, la cual constituye la esencia de éstos y no es otra cosa sino la divinidad, se predica tanto por separado como conjuntamente.

47. En efecto, se puede afirmar en primer lugar por separado: EL PADRE ES VERDAD, o sea, es verdadero. También El HiJo ES VERDAD, es decir, es verdadero. También El EsPíRITU SANTO ES VERDAD, o, lo que es lo mismo, es verdadero. En segundo lugar, también conjuntamente: EL PADRE, El HiJo y El ESPíritu SANTO NO SOn TRES VERDADES SINO son UNA singular y absoluta VERDAD, o sea, un solo ser verdadero. Así, debió asignar el plural del término a la pluralidad de las personas y decir «son». Sin embargo, a causa de la singularidad de la verdad en el predicado, dijo «ES» en singular.

48. He aquí que por este ejemplo resulta manifiesto que la verdad predicada de cada uno por separado a la vez se dice de los mismos conjuntamente. Así PUES, SI UNA SOLA VERDAD perteneciente a ellos, EN ELLOS ES - tal como efectivamente es - UNA SOLA SUSTANCIA, o sea, esencia, ES NECESARIO QUE LA misma VERDAD SE PREDIQUE SUSTANCIALMENTE de ellos. [H173] También SOBRE LA BONDAD así COMO SOBRE LA INCONMUTABILIDAD, SOBRE LA JUSTICIA, LA OMNIPOTENCIA Y SOBRE TODOS LOS RESTANTES PREDICADOS QUE HACEMOS EN SINGULAR TANTO DE CADA UNO TOMadO por separado COMO a la vez DE TODOS ELLOS - completamos nosotros:- decimos lo mismo que se dijo sobre la deidad y la verdad, a saber, que si son - como efectivamente son - una sustancia perteneciente a estos tres, ES EVIDENTE QUE HA DE PREDICARSE sobre ellos DE MODO SUSTANCIAL, pues se habla de dicha sustancia bajo una diversidad de muchos nombres.

49. Según esto, etc.

Aquí confirmó por la autoridad de la fe católica aquella parte de su razonamiento en la que se dice: «Cualquier cosa que se predique sustancialmente de Dios, es decir, del Padre, del Hijo y del Espíritu santo, se dice en singular de esos sujetos tanto por separado como a la vez».

50. Se dispone a asumir que ninguno de los tres acerca de los cuales existen denominaciones, o sea, «Padre», «Hijo» y «Espíritu santo», ni por separado ni a la vez se dicen de todos. [1308] Asimismo, se dispone a concluir que, «por consiguiente, ninguno de ellos se predica sustancialmente» y esto lo demostrará respecto de cada uno. Ahora, principiando por callar estos nombres - es decir, «Padre», «Hijo» y «Espíritu santo»-, afirma en general: AQUELLO y QUE en cuya generalidad se contienen éstos como especies.

51. Así, pues, como si introdujese brevemente en su razonamiento el lugar de la presuposición y de la conclusión, habla en general diciendo: SEGún ESTO, SE VE QUE AQUELLO...

Es como si dijese: la deidad, la verdad, la bondad y cualquier otra cosa que se predique sustancialmente de los tres, se dirá respecto de todos esos sujetos tanto por separado como conjuntamente. SEgÚn ESTO, SE VE QUE AQUELLO es en general cualquier cosa QUE CONVIENE SEA DICHA EN CADA UNO POR SEPARADO, NO SE PUEDE EMPERO DECIR EN TODOS igualmente con un término singular, NO SE PREDiCA SUSTANCIALMENTE, Sino más bien DE OTRO MODO. Más TARde INVESTIGARÉ CUÁL modo SEA ÉSTE.

52. EN EFECTO, QUIEN, etc.

Como si dijese: es verdad. Cualquier cosa que se diga de cualquiera de estos tres expresada por separado no se predica de todos ellos con un nombre singular, no se dice sustancialmente [H174] de ellos. EN EFECTO, estos términos —o sea, «Padre», «Hijo»y «Espíritu santo»—, que se predican de cada uno pero no de todos, no se dicen sustancialmente de todos en modo alguno. 
53. Esto es cierto sin ninguna ambigüedad. Efectivamente, QUIEN ES PADRE NO TRANSMITE TAL VOCABLO - por el cual Él se denomina «Padre»— AL HiJo Ni AL EsPíRITU SANTO, como si quien es Hijo o quien es Espíritu santo fueran denominados con el mismo término «Padre».

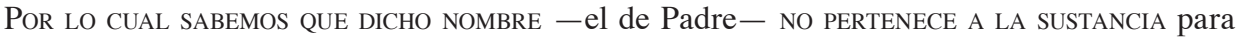
aquél solo que es llamado «Padre».

54. En otras palabras: la cosa aquí predicada con este nombre no pertenece a aquello de lo cual es predicado, la sustancia. Ciertamente, PUES SI FUERA DE CARÁCTER SUSTANCIAL, EL NOMBRE, es decir la cosa que ahí se predica, TAL COMO SUCEDE CON «Dios», es decir, la deidad, «VERDAD», «JUSTICIA» Y EL NOMBRE MISMO DE «SUSTANCIA», es decir, usya que referimos nosotros con el mencionado nombre de sustancia, TAMBIÉN SE DIRÍA DE LOS OTROS, o sea, del Hijo y del Espíritu santo.

55. Igualmente, tan SÓlo El Hijo Recibe tal nOMBre, a saber, el de «Hijo». En efecto, NI lo unE A LOS OTROS, es decir, al «Padre» y al «Espíritu santo». Esto significa que, ni aquel que es Padre, ni quien es Espíritu santo, son denominados con el nombre de «Hijo». IguALmENTE Dios, es decir, este nombre que es «Dios». Igualmente Verdad, o sea, este nombre que es «verdad». IGUALMENTE LOS DEMÁs nombres A LOS QUE ME REFERÍ ANTES con los cuales, sea por separado, sea conjuntamente, se predica de tres sujetos una sola usya, la cual es significada por dichos nombres.

56. Ciertamente, con el Padre y el Hijo, el «Espíritu» no sólo recibe AsIMISMo dicho nombre en virtud de la usya, sino también es denominado «santo» por el ejercicio de su capacidad de obrar - la cual es igual para los tres - o por su eternidad - por la cual son siempre aquello que son en virtud de su usya -; sin embargo, es denominado «ESPíRITU SANTO», reduciendo las significaciones de dichos nombres a la propiedad por la cual es entendido como distinto del Padre y el Hijo, pues no es el mismo Que el PAdRE y el HiJo.

57. Más adelante, concluye su raciocinio y dice: POR ESTO, es decir, es así que lo predicado sustancialmente acerca de Dios, también se dice en singular de aquellos sujetos, tanto por separado cuanto a la vez, es decir, del Padre, del Hijo y del Espíritu santo; no obstante, ni «Padre», ni «Hijo», ni «Espíritu [H175] santo» se dicen de todos ellos ni por separado ni conjuntamente. Por consiguiente, ENTENDEMOS correctamente QUE «PADRE», «HIJO» Y «ESPíRITU SANTO», o sea, los referidos con dichas voces, NO SE PREDICAN DE LA DIVINIDAD MISMA, es decir, de aquellas cosas que únicamente competen a la divinidad, además de «Dios», también se denominan «divinidad»; SINO más bien entendemos que se dicen DE OTRO MODO que ya se expondrá.

58. Si...

Es como si dijese: hemos mostrado adecuadamente que tales cosas no se dicen sustancialmente debido a que no se predican de todos los sujetos -Padre, Hijo y Espíritu santo- ni por separado ni a la vez. Si cualesquiera de ellos SE PREDICASEN DE MODO SUSTANCIAL, sin duda TAMBIÉN SE REFERIRÍAN TANTO A CADA UNO por separado COMO A TODOS los sujetos a la vez EN SINGULAR, porque la sustancia perteneciente a todos, por la cual son, es únicamente una.

\section{II}

\section{Pero es EVidente...}

Dijo que tales predicados no se hacían sustancialmente sino de otro modo. Ahora elucidará cuál sea este modo [1309] diciendo: Pero es EVIDENTE QUe ESTOS NOMBres SE DICEN EN RELACIÓN A ALGUIEN, es decir, según lo predicado por tales nombres, aquellos de los cuales se predica y esto mismo que son en virtud de tales predicados pertenecen a otros. 
No cabe duda, pues el Padre es padre de alguien, es decir, del Hijo, y al revés el Hijo es hiJo también de Alguien, es decir, es el Hijo del Padre y el Espíritu es espíritu de Alguien, o sea, del Padre y del Hijo, pues también del Hijo es Espíritu, o sea conexión.

60. De esto Resulta, etc.

Aquí debemos recordar, al parecer, que la unidad de todas las cosas diversas entre sí está asociada en cierto género de las facultades de los predicamentos. En efecto, siempre que se predica algo de cualquier cosa, este predicado es ciertamente aquello que significa el ser en virtud del nombre ínsito por el mismo y en virtud de la composición del verbo sustantivo con cuya añadidura es predicado.

61. [H176] Sin embargo, es uno en la unidad concomitante: tal como lo blanco es blanco en virtud de la blancura pero es uno gracias a la unidad concomitante a la blancura. Gracias a la blancura y, contemporáneamente, a su compañera la unidad, lo blanco es uno. Por esto, cuando se hacen muchos predicados sobre una sola cosa, en cierto modo, aquello que es uno, es muchos, porque es en virtud de muchos.

62. Aunque no hay un número para lo que es merced a muchos, existe empero un número para aquéllos, concretamente, uno para cada uno de ellos. Asimismo, existe para las unidades de lo concomitante a ellos: para cada una de las cuales también hay un número.

63. Puesto que, por otra parte, una cosa se predica de muchos sujetos, los muchos se vuelven uno, mientras que, cuando se dicen muchas cosas de muchos sujetos, los muchos son muchos. En efecto, según el número de aquellos que se predican, también pertenece a los «unos» el número de aquellas unidades concomitantes, debido a cierta conformidad. Por fin, la unidad carece de número cuando no existe número alguno para aquel predicado correspondiente a tal «uno» con el cual es concomitante.

64. Así pues, la paternidad, la filiación y la conexión son distintas, luego también las unidades presentes a éstas han de ser distintas entre sí. Dado que, aunque a una sustancia le puede acompañar otra sustancia o un accidente - como la animación y el color acompañan a la corporalidad-, pero a un accidente no puede acompañarle una sustancia, entonces, las unidades que acompañan a la paternidad, a la filiación y a la conexión nunca podrán ser sustancias. Estas unidades serán tres, no únicamente para estos predicados (paternidad, filiación y conexión) sino también para aquéllos de los cuales se predican, o sea, el Padre, el Hijo y el Espíritu santo. Por este motivo, el autor infiere correctamente cuando dice: DE ESTO RESULTA QUE TAMPOCO SE PREDICA DE Dios LA TRINIDAD DE MODO SUSTANCIAL.

65. [H177] También resulta claro por ello que no se dice «trinidad» por separado de cada uno de ellos - el Padre, el Hijo y el Espíritu santo-: En efecto, El PADRE NO es TRINIDAD, es decir, el Padre no es los tres. Sin duda. Aquel Que es PAdre es ciertamente Padre, pero no ES HiJo Ni EsPíRitu SANTo. Es necesario aprobar tales cosas enteramente.

66. En efecto, las unidades acompañan únicamente a aquello que se predica, pero no lo hacen sino en cuanto los predicados se pueden efectuar de ciertos sujetos. Por consiguiente, es imposible predicarlas mientras falten aquéllos a los cuales han de acompañar. Así, no puede ser un Hijo quien no es Hijo, ni ser un Espíritu santo quien no es Espíritu santo.

67. Del mismo modo, tampoco el Hijo es trinidad, es decir, no es los tres porque, quien es Hijo, es el Hijo, pero no es el Padre ni el Espíritu santo. Ni El Espíritu SANTO ES TRINIDAD, es decir, no es los tres porque, quien es Espíritu santo, o sea, Conexión, posee tal nombre propio. Pero no recibe los otros dos, vale decir, no es el Padre ni el Hijo.

68. Puesto que a los tres compete una sola divinidad, es necesario que los que, en virtud de ella, son Dios, también sean uno en virtud de la unidad que se produce según aquélla. Así, 
son un Dios merced a la divinidad y, contemporáneamente, a aquella unidad que se produce en virtud de ella.

El Padre, el Hijo y el Espíritu santo son, por consiguiente, a la vez tres y uno.

69. Por El CONTRARio, como se dijo, LA TRINIDAd CONSISTE EN LA PLURALIDAD DE PERSONAS. Es decir: el número de las unidades se predica acerca de ellos según [H178] el número de las propiedades, las cuales son también diversas entre sí y por eso se predican acerca de cosas distintas; en efecto, no se hacen nunca tales predicados unitariamente acerca de algo que está en la plenitud de su propiedad, la cual se reúne a partir de estos predicamentos y de todos los otros. Por ende, cualquiera de ellos son uno por sí, en virtud de su propiedad que poseen en relación a otro. En otras palabras, cada uno constituye una persona distinta.

70. LA UNIDAD de ellos, EN CAMBIO, consiste EN LA SIMPLICIDAD DE LA SUSTANCIA, es decir, de la usya, la cual se predica de ellos. Es decir, la unidad se predica de ellos [1310] según su esencia singular y simple, por la cual son lo que son.

71. Así, si, etc.

En este punto, dice lo mismo, a saber, que lo no común a los tres - al Padre, al Hijo y al Espíritu santo - no se predica sustancialmente en modo alguno. Por el contrario, lo perteneciente a la sustancia se dice de ellos en común.

Es como si dijese que la Trinidad consiste en la diversidad de personas, pero la unidad en la simplicidad de la sustancia. Si el Padre, el Hijo y el Espíritu santo son personas distintas - pues son sin excepción en virtud de las propiedades que no pueden decirse de uno-, PERO LA SUSTANCIA, o sea, usya, por la cual son lo que son, ES INDIVISA, entonces ES NECESARIO QUE EL TÉRMINO QUE TOMA SU ORIGEN DE LAS PERSONAS, NO PERTENEZCA A LA SUSTANCIA. Este término se enuncia apoyándose en la propiedad personal, sea propia de uno (como «Padre»), sea propia de dos (como «procedente», que no se dice del Padre sino del Hijo y del Espíritu santo; también es doble «emitente», que no se dice del Espíritu santo pero sí del Padre y del Hijo), sea propia de todos (como «tres» que no se dice de ninguno por separado sino de los tres conjuntamente). Por fin, que No PERTENEZCA A LA SUSTANCia significa que en absoluto se predica sustancialmente de aquella esencia por la cual son.

72. Sin EMBARGO, LA TRINIDAD, es decir, las unidades que, una vez reunidas, tres pueden ser enumeradas, PRODUCE LA DISTINCIÓN de las propiedades a partir de las cuales existe una distinción que se denomina distinción DE LAS PERSONAS. Puesto que, POR CONSIGUIENTE, aquellas propiedades no pertenecen a la sustancia (algo del todo cierto por lo que acabamos de ver, pues no se predican en singular de todos los sujetos por separado ni conjuntamente), luego con mayor razón LA TRINIDAD NO PERTENECE A LA SUSTANCIA, es decir, no es sustancial: evidentemente, no son sustanciales las unidades por las cuales se enumeran las propiedades junto a aquéllos a quienes pertenecen. En efecto, ni se predican todas a la vez ni por separado de cada uno.

73. [H179] Por decir lo mismo de otra manera: se trata de una certísima regla aquélla por la cual se afirma que no es sustancia lo predicado, por separado y conjuntamente, de los tres sujetos.

74. De esto resulta que ni el Padre, ni el Hijo, ni el Espíritu santo, es decir, cualquiera de aquellas propiedades por las cuales poseen tales nombres, NI LA TRINIDAD, por la cual y en virtud de aquéllas, el Padre, el Hijo y el Espíritu santo son tres, SE DICEN DE Dios (o sea, del Padre, del Hijo y del Espíritu santo) Sustancialmente, Sino - COMO arriba SE DIJO- EN RELACIÓN A ALGUIEN porque, ciertamente, el Padre y el Hijo están relacionados entre sí por la paternidad y la filiación y el Espíritu santo se encuentra en conexión respecto de ellos.

75. La Trinidad, por su parte, en virtud de la cual se dice de ellos «tres», no es empero una relación, pues todo número es por sí mismo, sino que acompaña a las relaciones con las cuales 
se encuentran vinculados. Por tanto, la asociación de aquéllas se denomina «predicación en relación a alguien».

76. «DIOS» EMPERO — es decir, la divinidad entendida por dicho nombre-, ASÍ COMO «VERDAD», «BONDAD», «OMNIPOTENCIA», «SUSTANCIA», «INMUTABILIDAD», «PODER», «SABIDURÍA» Y TODO LO QUE DE TAL MODO PUEDA PENSARSE - o sea, lo distinto por el nombre pero idéntico en la realidad- SE Dice sustancialmente De la divinidad, esto es, del Padre, del Hijo y del Espíritu santo. En efecto, en virtud de una sola $u s y a$, se dice de aquello que ellos son, sea por separado sea conjuntamente, que es «Dios, verdadero, justo, bueno, omnipotente, subsistente, inmutable, fuerte, sabio» y otros nombres de este tipo, tomados todos de la misma usya.

77. Te RUEGo ME AClares Si, etc.

Como si dijera: merced a los razonamientos antedichos cuyo punto de partida se halla en la fe católica, me parece que se ha demostrado que el Padre, el Hijo y el Espíritu santo no se predican sustancialmente de la divinidad. Ahora bien, oh Juan, aguardo tu juicio sobre ellos.

78. De tal modo, TE RUEGO ME ACLARES con la autoridad de tu respuesta SI TODO ESTO ESTÁ DICHO CORRECTAMENTE concordando con los lugares propios de las razones convenientes a las cuestiones teológicas y si, además, ha sido pronunciado SEGÚN LA FE, vale decir, de acuerdo con la fe católica; [H180] en otras palabras, te pido que me confirmes en la inteligencia de todo lo dicho hasta aquí.

79. O BIEN, SI, disintiendo de mí, OPINAS DE MODO DIVERSO EN ALGÚN ASPECTO, es decir, respecto de algo de lo antedicho, te suplico no eches a perder la razón por una fe fingida ni la fe por una razón artificiosa. Por el contrario, te solicito EXAMINES ATENTAMENTE - es decir, con gran diligencia - si Lo DICHO, ya pertenece a la fe católica, ya se sigue de lo que la fe católica sostiene.

80. Finalmente, te insto a que PONGAS DE ACUERDo LA FE y LA RAZÓn EN LA MEDIDA DE LO POSIBLE, de tal manera que, en primer lugar, concedas autoridad a la razón gracias a la fe, para después comparar el asentimiento de la fe desde el punto de vista de la razón. 